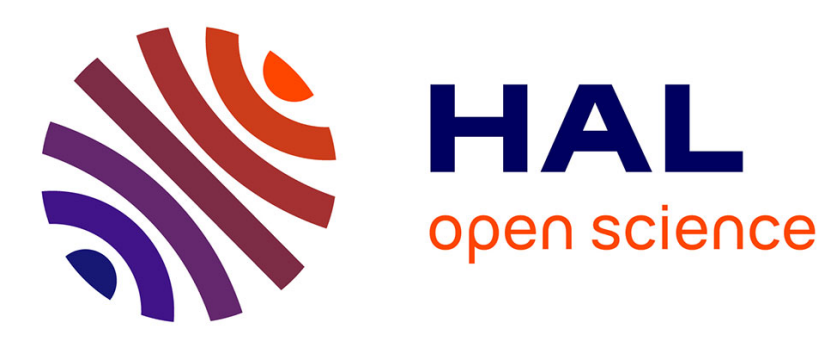

\title{
The excitation of $\mathrm{OH}$ by $\mathrm{H} 2$ revisited - II. Hyperfine resolved rate coefficients
}

J Klos, P Dagdigian, M Alexander, A Faure, François Lique

\section{To cite this version:}

J Klos, P Dagdigian, M Alexander, A Faure, François Lique. The excitation of OH by H2 revisited II. Hyperfine resolved rate coefficients. Monthly Notices of the Royal Astronomical Society, 2020, 493

(3), pp.3491-3495. 10.1093/mnras/staa530 . hal-03044757

\section{HAL Id: hal-03044757 \\ https://hal.science/hal-03044757}

Submitted on 18 Dec 2020

HAL is a multi-disciplinary open access archive for the deposit and dissemination of scientific research documents, whether they are published or not. The documents may come from teaching and research institutions in France or abroad, or from public or private research centers.
L'archive ouverte pluridisciplinaire HAL, est destinée au dépôt et à la diffusion de documents scientifiques de niveau recherche, publiés ou non, émanant des établissements d'enseignement et de recherche français ou étrangers, des laboratoires publics ou privés. 


\title{
The excitation of $\mathrm{OH}$ by $\mathrm{H}_{2}$ revisited - II. Hyperfine resolved rate coefficients
}

\author{
J. Kłos, ${ }^{1}$ P. J. Dagdigian, ${ }^{2}$ M. H. Alexander, ${ }^{1}$ A. Faure ${ }^{\oplus 3}$ and F. Lique ${ }^{1,4 \star}$ \\ ${ }^{1}$ Department of Chemistry and Biochemistry, University of Maryland, College Park, MD 20742-2021, USA \\ ${ }^{2}$ Department of Chemistry, The Johns Hopkins University, Baltimore, MD 21218-2685, USA \\ ${ }^{3}$ Université Grenoble Alpes, CNRS, IPAG, F-38000 Grenoble, France \\ ${ }^{4}$ LOMC - UMR 6294, CNRS-Université du Havre, 25 rue Philippe Lebon, BP 1123, F-76 063 Le Havre cedex, France
}

Accepted 2020 February 20. Received 2020 January 15; in original form 2020 January 10

\begin{abstract}
Observations of hyperfine resolved transitions of the hydroxyl radical $(\mathrm{OH})$ are unique probes of the physical conditions in molecular clouds. In particular, hyperfine intensities can be used as an effective thermometer over a wide range of molecular densities. Accurate modelling of the $\mathrm{OH}$ emission spectra requires the calculation of collisional rate coefficients for the excitation of $\mathrm{OH}$ by $\mathrm{H}_{2}$, the most abundant collisional partner in the molecular clouds. Here, we determine hyperfine resolved rate coefficients for the excitation of $\mathrm{OH}$ by $\mathrm{H}_{2}$ using a recently developed highly accurate potential energy surface. State-to-state rate coefficients between the lower hyperfine levels were calculated using recoupling techniques for temperature ranging from 10 to $150 \mathrm{~K}$. Significant differences were found with the earlier values currently used in astrophysical models, the new rate coefficients being larger than the previous ones. Finally, we compute the excitation of the $\mathrm{OH}$ radical in cold molecular clouds and star-forming regions. The new rate coefficients were found to increase the hyperfine intensities by a factor of $\sim 1-2$. Consequently, we recommend using this new set of data in any astrophysical model of $\mathrm{OH}$ excitation.
\end{abstract}

Key words: molecular data-molecular processes - ISM: abundances.

\section{INTRODUCTION}

The rotational transitions of the hydroxyl radical $(\mathrm{OH})$ are unique probes of diffuse and dense interstellar clouds, late-type stars, and external galaxies (e.g. Harju, Winnberg \& Wouterloot 2000; Goicoechea et al. 2011; Cotten et al. 2012; Wampfler et al. 2013; Ebisawa et al. 2015). Indeed, observations of OH provide critical constraints on both the density and temperature of the media. Moreover, the $\mathrm{OH}$ radical is a key species in the oxygen and water chemistry network, because its presence is tightly related to the formation and destruction of water, and modelling the abundance of $\mathrm{OH}$ in different media is of fundamental interest.

In addition, in many interstellar sources, the hyperfine structure of $\mathrm{OH}$ transitions is resolved (e.g. Harju et al. 2000; Wampfler et al. 2011; Ebisawa et al. 2015; Ebisawa et al. 2019). Indeed, due to the nuclear spin of the proton, the $\mathrm{OH}$ rotational levels split into hyperfine sublevels. Resolving the hyperfine structure of a rotational transition is extremely useful because, in principle, the optical depth of the transition can be obtained directly by comparing the relative intensities of the hyperfine lines. By assuming that all components have the same linewidth and excitation temperature, a simultaneous fit of all hyperfine components can be performed. This fit provides both the optical depth of each component and the excitation temperature.

However, the radio-frequency lines of $\mathrm{OH}$ in astronomical sources almost never show the expected intensity ratios. This results from the fact that the $\mathrm{OH}$ rotational transitions have very large radiative transition probabilities, which implies that the levels become thermalized at much higher densities than they normally occur in the interstellar medium (ISM). This is particularly true for the transition between $\Lambda$-doublet levels in the ground rotational state at $18 \mathrm{~cm}^{-1}$. Indeed, in the ISM, the relative population of the four hyperfine structure levels often deviates from the values expected from the single excitation temperature assumption. In extreme cases, some of these hyperfine component lines even display maser emission. It has been shown that the above anomaly of hyperfine intensities can be used as an effective thermometer of molecular clouds over a wide range of $\mathrm{H}_{2}$ densities (Ebisawa et al. 2015).

Hence, full exploitation of the $\mathrm{OH}$ observations relies on radiative transfer calculations that require the computation of collisional data. Collisional excitation rate coefficients for the $\mathrm{OH}$ radical are mandatory to derive precisely $\mathrm{OH}$ abundances in the ISM from the molecular spectra (Roueff \& Lique 2013). As a consequence, several calculations of rate coefficients for the $\mathrm{OH}$ molecule colliding with $\mathrm{He}$ and $\mathrm{H}_{2}$, the two dominant collisional partners 
in the ISM have been provided over the last decades. We refer to Kłos et al. (2017, hereafter, Paper I) for a brief review of the available calculations. We mention specifically Offer, van Hemert \& van Dishoeck (1994, hereafter OHD94), who computed hyperfine resolved rate coefficients for $\mathrm{OH}-\mathrm{H}_{2}$ collisions.

Recently, we employed the coupled-cluster potential energy surfaces (PESs) of Ma et al. (2014) to generate a new set of rate coefficients for the rotational excitation of $\mathrm{OH}$ through collisions with $\mathrm{H}_{2}$. In these calculations, we did not include the hyperfine structure. Then, in this paper, we extend the calculations of Paper I to the hyperfine structure in order to provide the astrophysical community with all the necessary data for extracting maximum information from the $\mathrm{OH}$ spectra.

This paper is organized as follows: Section 2 briefly describes the scattering calculations. In Section 3, we report state-to-state hyperfine resolved cross-sections and rate coefficients for the collisional excitation of $\mathrm{OH}$ by $\mathrm{H}_{2}$, and compare our calculated rate coefficients with previous $\mathrm{OH}-\mathrm{H}_{2}$ rates currently used for astrophysical modelling. In Section 4, we present the first astrophysical application of our newly calculated rate coefficients. Conclusions are given in Section 5 .

\section{THEORETICAL APPROACH}

${ }^{1}$ We have carried out exact time-independent quantum scattering calculations for the collision of an open-shell molecule in a ${ }^{2} \Pi$ electronic state with a closed-shell diatomic molecule, following the formalism presented previously (Offer \& Flower 1990; Offer et al. 1994; Groenenboom, Fishchuk \& van der Avoird 2009; Ma et al. 2014; Schewe et al. 2015a). Our calculations were performed with the HIBRIDON (Hibridon 2012) suite of scattering programs using the ${ }^{2} \Pi-\Sigma^{+}$molecule-molecule basis. The $\mathrm{OH}$ and $\mathrm{H}_{2}$ molecules were taken to be rigid, with their internuclear separations fixed to the average values in their ground vibrational levels.

The $\mathrm{OH}\left({ }^{2} \Pi\right)$ state is split into two fine-structure manifolds, labelled $F_{1}$ and $F_{2}$ for the lower and upper set of levels, respectively (see Huber \& Herzberg 1979). For low values of the OH total angular momentum $j$, these correspond to molecule-fixed projections $\Omega=$ $3 / 2$ and $1 / 2$, respectively. The rotational levels come in closely spaced pairs, namely the $\Lambda$-doublets, of opposite parity + and - . The parity defines the action of the space-fixed inversion operator on the molecular wavefunction: $i^{\mathrm{SF}} \psi=p \psi$. In this work, we denote the $\mathrm{OH}$ rotational levels by the total angular momentum $j$, the finestructure label $F_{i}(i=1,2)$, and the parity $p$.

The channel energies were computed using $\mathrm{OH}$ and $\mathrm{H}_{2}$ spectroscopic constants given in Paper I. The scattering calculations were performed using the $\mathrm{OH}-\mathrm{H}_{2}$ RCCSD(T)-F12 PESs of Ma et al. (2014). The grid of collision energies, $\mathrm{OH}$ and $\mathrm{H}_{2}$ channel basis, range of total angular momenta $J$, and integration parameters are the same as were employed in Paper I. Our calculations were found to be converged, as described in Paper I.

The hyperfine resolved cross-sections were determined from the nuclear-spin-free $T$-matrix elements (Offer et al. 1994). We denote the total angular momentum of the collision complex by $J_{T}=J$ $+I$, where $J$ is the total angular momentum of the complex in the absence of the nuclear spin and $I$ is the nuclear spin of the proton. The total angular momentum of the $\mathrm{OH}$ radical, including nuclear spin, is denoted by $F=j+I$, and the rotational angular momentum $j_{2}$ of the $\mathrm{H}_{2}$ collision partner equals 0 and 1 for para- and ortho- $\mathrm{H}_{2}$, respectively. The $T$-matrix elements describing the collision of two molecules with angular momenta $j$ and $j_{2}$, including nuclear spin, can be computed from the nuclear-spin-free $T$-matrix elements:

$$
\begin{aligned}
T_{j^{\prime} F_{i}^{\prime} p^{\prime} F^{\prime} j_{2}^{\prime} j_{R}^{\prime} l^{\prime}, j F_{i} p F j_{2} j_{R} l}^{J_{T}}= & \sum_{J_{12}^{\prime} j_{12}}(-1)^{j_{R}+j_{R}^{\prime}+l+l^{\prime}+j_{2}+j_{2}^{\prime}} \\
& \times\left([F]\left[F^{\prime}\right]\left[j_{12}\right]\left[j_{12}^{\prime}\right]\left[j_{R}\right]\left[j_{R}^{\prime}\right]\right)^{1 / 2}(2 J+1) \\
& \times\left\{\begin{array}{lll}
j & j_{2} & j_{12} \\
l & J & j_{R}
\end{array}\right\}\left\{\begin{array}{lll}
j^{\prime} & j_{2}^{\prime} & j_{12}^{\prime} \\
l^{\prime} & J & j_{R}^{\prime}
\end{array}\right\} \\
& \times\left\{\begin{array}{lll}
j_{R} & j & J \\
I & J_{T} & F
\end{array}\right\}\left\{\begin{array}{ccc}
j_{R}^{\prime} & j^{\prime} & J \\
I & J_{T} & F^{\prime}
\end{array}\right\} \\
& \times T_{j^{\prime} F_{i}^{\prime} p^{\prime} j_{2}^{\prime} j_{12}^{\prime} l^{\prime}, j F_{i} p j_{2} j_{12} l,}
\end{aligned}
$$

where $[x]=2 x+1$. The quantum numbers $l, j_{12}$, and $j_{R}$ denote the orbital angular momentum of the collision complex, the vector sum of $j+j_{2}$, and the vector sum of $j_{2}+l$, respectively. The initial and final levels are indicated by unprimed and primed quantum numbers, respectively.

Integral cross-sections for hyperfine resolved transitions can be calculated from the $T$-matrix elements in equation (1) as follows:

$$
\begin{aligned}
\sigma_{j^{\prime} F_{i}^{\prime} p^{\prime} F^{\prime} j_{2}^{\prime}, j F_{i} p F j_{2}}= & \frac{\pi}{k_{i}^{2}(2 F+1)\left(2 j_{2}+1\right)} \sum_{J_{T}}\left(2 J_{T}+1\right) \\
& \times \sum_{l l^{\prime} j_{R} j_{R}^{\prime}}\left|T_{j^{\prime} F_{i}^{\prime} p^{\prime} F^{\prime} j_{2}^{\prime} j_{R}^{\prime} l^{\prime}, j F_{i} p F j_{2} j_{R} l}^{J_{T}} l\right|^{2} .
\end{aligned}
$$

The corresponding rate coefficients are obtained by taking the thermal average of the cross-section (equation 2) over the collision energy $\left(E_{\mathrm{c}}\right)$

$k_{i \rightarrow f}(T)=\left(\frac{8}{\pi \mu k_{\mathrm{B}}^{3} T^{3}}\right)^{1 / 2} \int_{0}^{\infty} \sigma_{i \rightarrow f}\left(E_{\mathrm{c}}\right) E_{\mathrm{c}} \exp \left(-\frac{E_{\mathrm{c}}}{k_{\mathrm{B}} T}\right) \mathrm{d} E_{\mathrm{c}}$.

\section{RESULTS}

\subsection{Rate coefficients}

Hyperfine resolved state-to-state integral cross-sections were computed for 24 initial $\mathrm{OH}$ rotational/hyperfine levels up to the $X^{2} \Pi_{1 / 2}$, $j=5 / 2, p=+/-$ levels (with energy equal to $289 \mathrm{~cm}^{-1}$ ) in collisions with para $-\mathrm{H}_{2}$ and ortho $-\mathrm{H}_{2}$, in their $j_{2}=0$ and $j_{2}=1$ levels, respectively. The corresponding rate coefficients were computed for the temperature range from 10 to $150 \mathrm{~K}$.

Fig. 1 presents rate coefficients for transitions between the $\Lambda$ doublets associated with the $\mathrm{OH}$ ground $j=3 / 2 F_{1}$ rotational level induced by collisions with para $-\mathrm{H}_{2}\left(j_{2}=0\right)$ and ortho- $\mathrm{H}_{2}\left(j_{2}=\right.$ 1). Plotted in panel (a) are transitions with $\Delta F=\Delta j$, while panel (b) displays rate coefficients with $\Delta F \neq \Delta j$. Comparing the rate coefficients in the two panels, we see that these display the expected propensity for $\Delta F=\Delta j$ transitions (Alexander \& Dagdigian 1985).

The rate coefficients for the transitions between the $j=$ $3 / 2 F_{1} \Lambda$-doublet levels are seen to be much larger for collisions with ortho $-\mathrm{H}_{2}\left(j_{2}=1\right)$ than with para- $\mathrm{H}_{2}\left(j_{2}=0\right)$. This propensity was previously observed in cross-sections and rate coefficients for transitions between $\mathrm{OH}$ rotational/fine-structure levels (Schewe et al. 2015a; Paper I). The strong anisotropic interaction between the $\mathrm{OH}$ dipole moment and the $\mathrm{H}_{2}$ quadrupole moment is responsible for the large cross-sections, involving collisions with ortho- $\mathrm{H}_{2}\left(j_{2}=\right.$ $1)$. By contrast, the $\mathrm{H}_{2}\left(j_{2}=0\right)$ level is spherical and, hence, has no 


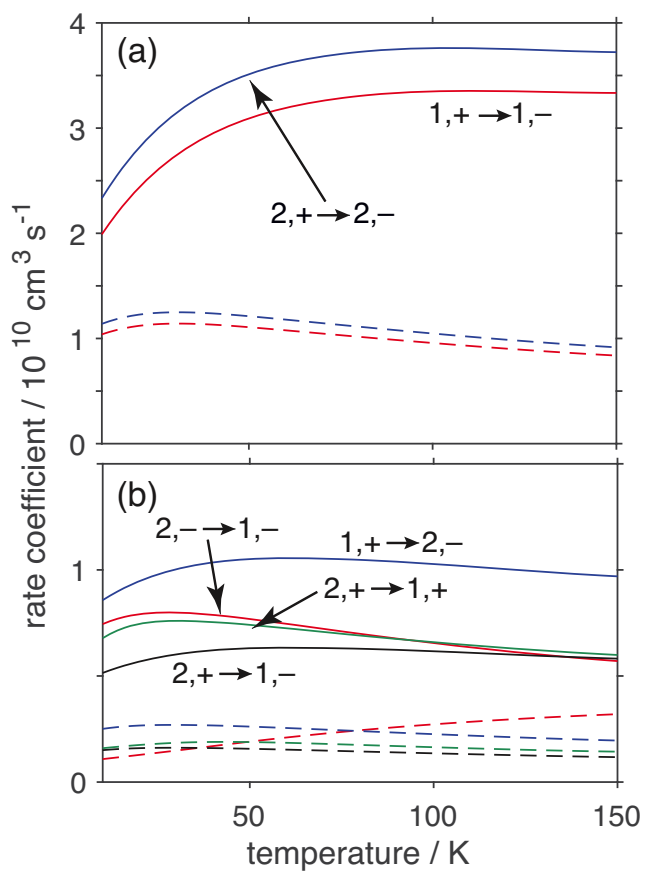

Figure 1. Rate coefficients as a function of temperature for de-excitation transitions between the $\Lambda$-doublets of the $\mathrm{OH}$ ground $j=3 / 2 F_{1}$ rotational/fine-structure level. (a) Transitions with $\Delta F=\Delta j$, and (b) transitions with $\Delta F \neq \Delta j$. Rate coefficients plotted with solid and dashed lines are for collisions with ortho $-\mathrm{H}_{2}\left(j_{2}=1\right)$ and para $-\mathrm{H}_{2}\left(j_{2}=0\right)$, respectively. The levels are labelled by the total angular momentum $F$ and the parity $p$ for transitions involving collisions with ortho- $\mathrm{H}_{2}\left(j_{2}=1\right)$; a similar colour coding applies to transitions involving collisions with para- $\mathrm{H}_{2}\left(j_{2}=\right.$ $0)$.

quadrupole moment. Hence, the cross-sections and rate coefficients for this collision partner are much smaller.

We display in Fig. 2 rate coefficients for fine-structure conserving and fine-structure changing rotational transitions, namely $j=5 / 2$ $F_{1} \rightarrow 3 / 2 F_{1}$ and $j=1 / 2 F_{2} \rightarrow 3 / 2 F_{1}$ transitions, respectively. The rate coefficients for the former can be seen to be slightly larger than for the latter. In general, rate coefficients for collisions with ortho$\mathrm{H}_{2}\left(j_{2}=1\right)$ are larger than for collisions with para- $\mathrm{H}_{2}\left(j_{2}=0\right)$, as explained above. However, unlike the case of the rate coefficients for the $\Lambda$-doublet transitions plotted in Fig. 1, this is not universal. Rate coefficients for $\Delta F \neq \Delta j$ are not plotted since these are much smaller than for $\Delta F=\Delta j$ transitions.

As noted previously concerning the fine-structure resolved crosssections and rates (Schewe et al. 2015a; Paper I) and seen in the data plotted in Figs 1 and 2, the hyperfine resolved rate coefficients display a dependence on the parities of both the initial and final levels.

\subsection{Comparison with previous results}

A widely employed data set of $\mathrm{OH}-\mathrm{H}_{2}$ rate coefficients in radiative transfer calculations is that of Offer et al. (1994). Fig. 3 compares the rate coefficients computed by Offer et al. (1994) and in this work for all de-excitation transitions between the 24 lowest $\mathrm{OH}\left(X^{2} \Pi\right)$ hyperfine levels in collisions with both para- $\mathrm{H}_{2}\left(j_{2}=0\right)$ and ortho$\mathrm{H}_{2}\left(j_{2}=1\right)$.

There are noticeable differences in the magnitudes of the rate coefficients from the two sets of calculations, as was also previ-

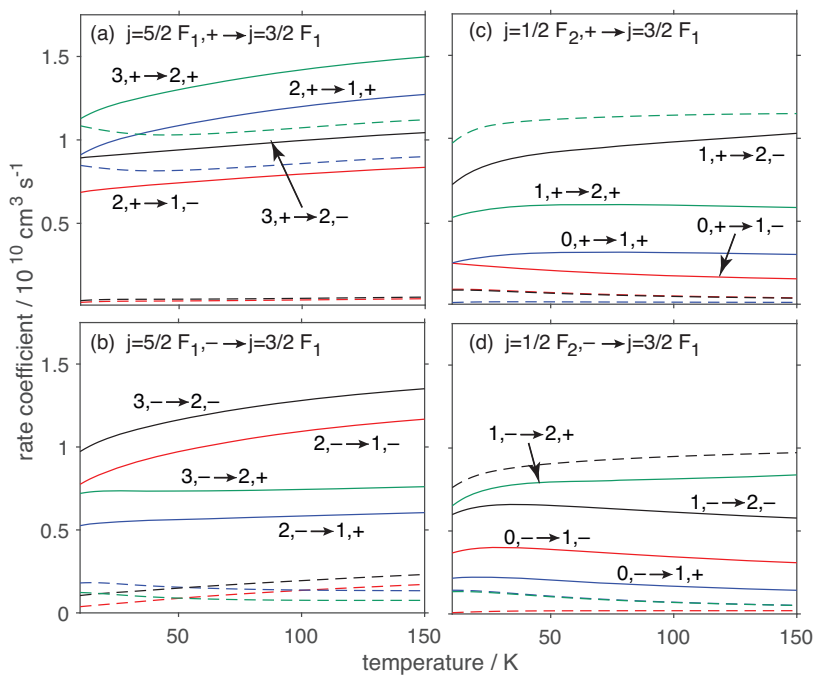

Figure 2. Rate coefficients as a function of temperature for fine-structure conserving and fine-structure changing de-excitation transitions to the ground $j=3 / 2 F_{1}$ manifold in collisions with ortho- $\mathrm{H}_{2}\left(j_{2}=1\right)$ (solid lines) and para $-\mathrm{H}_{2}\left(j_{2}=0\right)$ (dashed lines). (a) Fine-structure conserving transitions from the $j=5 / 2 F_{1}$ level of + parity, (b) fine-structure conserving transitions from the $j=5 / 2 F_{1}$ level of - parity, (c) fine-structure changing transitions from the $j=1 / 2 F_{2}$ level of + parity, and (d) fine-structure changing transitions from the $j=1 / 2 F_{2}$ level of - parity. For clarity, only the $\Delta F=\Delta j$ transitions are plotted. The levels are labelled by the total angular momentum $F$ and the parity $p$ for transitions involving collisions with ortho$\mathrm{H}_{2}\left(j_{2}=1\right)$; a similar colour coding applies to transitions involving collisions with para $-\mathrm{H}_{2}\left(j_{2}=0\right)$.

ously found in the comparison of the fine-structure resolved rate coefficients (Paper I). At a low temperature [see Fig. 3(a)], the new rate coefficients are slightly larger by a factor of 1.5-2, on average. However, the differences in the magnitudes of the rate coefficients depend strongly on the particular transition. For some transitions, the rate coefficient from Offer et al. (1994) is larger than the value computed in this work. At higher temperatures [see Fig. 3(b)], the differences between the two sets of rate coefficients are appreciably smaller. However, the rate coefficients from our new set are still slightly larger, on average.

The differences in the two sets of rate coefficients can be explained by differences in the PESs, which were employed in the scattering calculations. The overall dependence of the two PESs on the intermolecular separation and orientations is similar. However, the well depth of the PES employed in the present calculations, $\sim 220 \mathrm{~cm}^{-1}$ (Ma et al. 2014), is greater than for the PES (Offer \& van Hemert 1993) employed by Offer et al. (1994). This leads to slightly different shapes of the PESs and, hence, different magnitudes of the cross-sections and rate coefficients for inelastic transitions.

The high accuracy of the PES used in this work and, hence, the accuracy of the inelastic rate coefficients was validated by a comparison of cross-sections measured in the crossed beam experiments of Schewe et al. (2015b). Hence, we recommend the use of the present set of rate coefficients in astrophysical applications.

\section{ASTROPHYSICAL APPLICATIONS}

As an application of our new rate coefficients, we have performed radiative transfer calculations to simulate the hyperfine excitation of $\mathrm{OH}$ in the ISM. Indeed, the hyperfine structure of $\mathrm{OH}$ transitions is resolved in many interstellar and circumstellar sources, and 


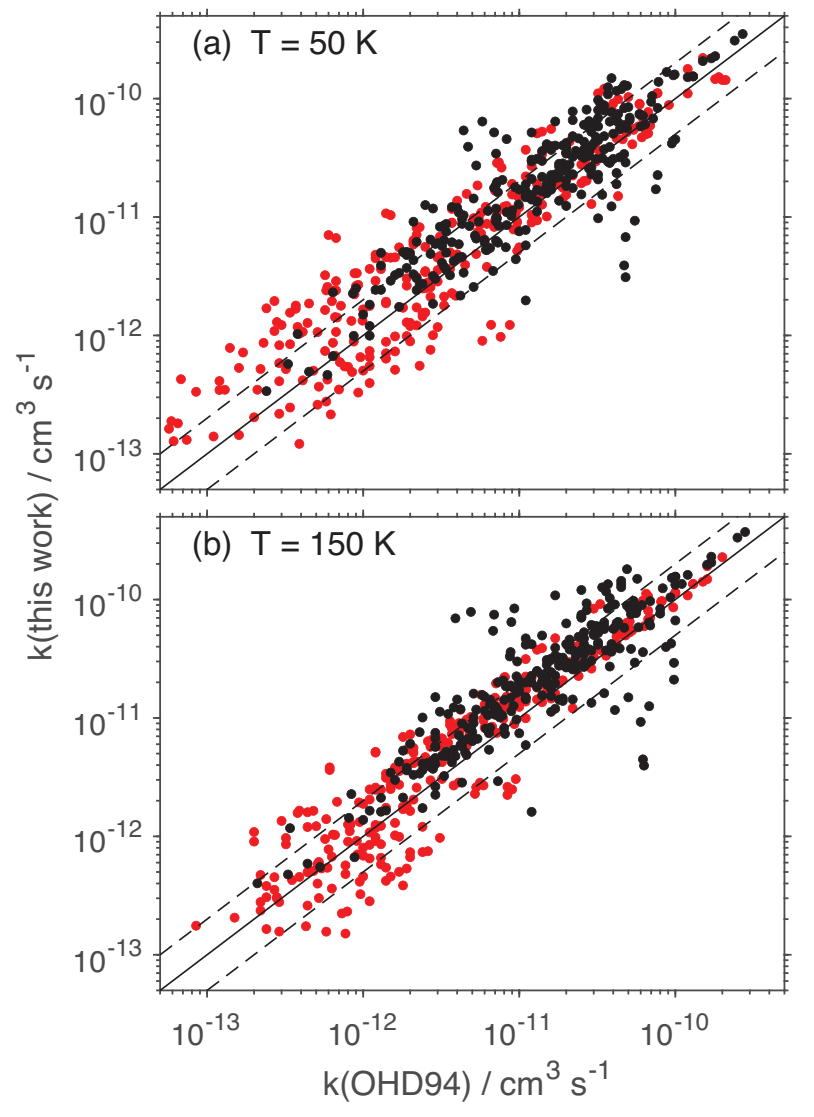

Figure 3. Comparison between rate coefficients computed by Offer et al. (1994) and in this work for all de-excitation transitions between the 24 lowest $\mathrm{OH}\left(X^{2} \Pi\right)$ hyperfine levels at a temperature of (a) $50 \mathrm{~K}$ and (b) $150 \mathrm{~K}$. The vertical axis represents $\mathrm{OH}-\mathrm{H}_{2}$ rate coefficients computed in this work, while the horizontal axis represents the corresponding rate coefficients from Offer et al. (1994). Rate coefficients for collisions with both para- $\mathrm{H}_{2}\left(j_{2}=\right.$ $0)$ (red circles) and ortho $-\mathrm{H}_{2}\left(j_{2}=1\right)$ (black circles) are displayed. The two dashed lines delineate the region in which the rate coefficients differ by a factor of less than 2 .

hyperfine intensities can be used as a tracer of physical conditions, as discussed in the Introduction. Recent examples include the work of Ebisawa et al. (2015, 2019), where detailed non-local thermodynamic equilibrium (non-LTE) calculations were performed using the collisional data of OHD94 to reproduce Effelsberg 100-m observations towards molecular clouds. Here, we do not intend to model specific observations but to assess the impact of the new hyperfine resolved rate coefficients on generic non-LTE results. We do not include, in particular, dust continuum radiation and we do not treat line overlaps.

Non-LTE radiative transfer calculations were performed with the RADEX code (van der Tak et al. 2007). The escape probability formalism approximation for an expanding sphere was used. We consider a uniform spherical geometry of the interstellar cloud. Both collisional and radiative processes are taken into account in the calculations, and we assume a cosmic microwave background $(\mathrm{CMB})$ radiation of $2.73 \mathrm{~K}$. In all the calculations, we consider a thermal distribution of the two nuclear spin isomers of $\mathrm{H}_{2}$.

First, we focus on the four hyperfine components of the $j=3 / 2$ $F_{1},+\rightarrow j=3 / 2 F_{1},-$ radiative transition at $1.66 \mathrm{GHz}$. Indeed, for these transitions between $\Lambda$-doublet levels in the ground rotational state at $18 \mathrm{~cm}^{-1}$, the relative populations of the four hyperfine
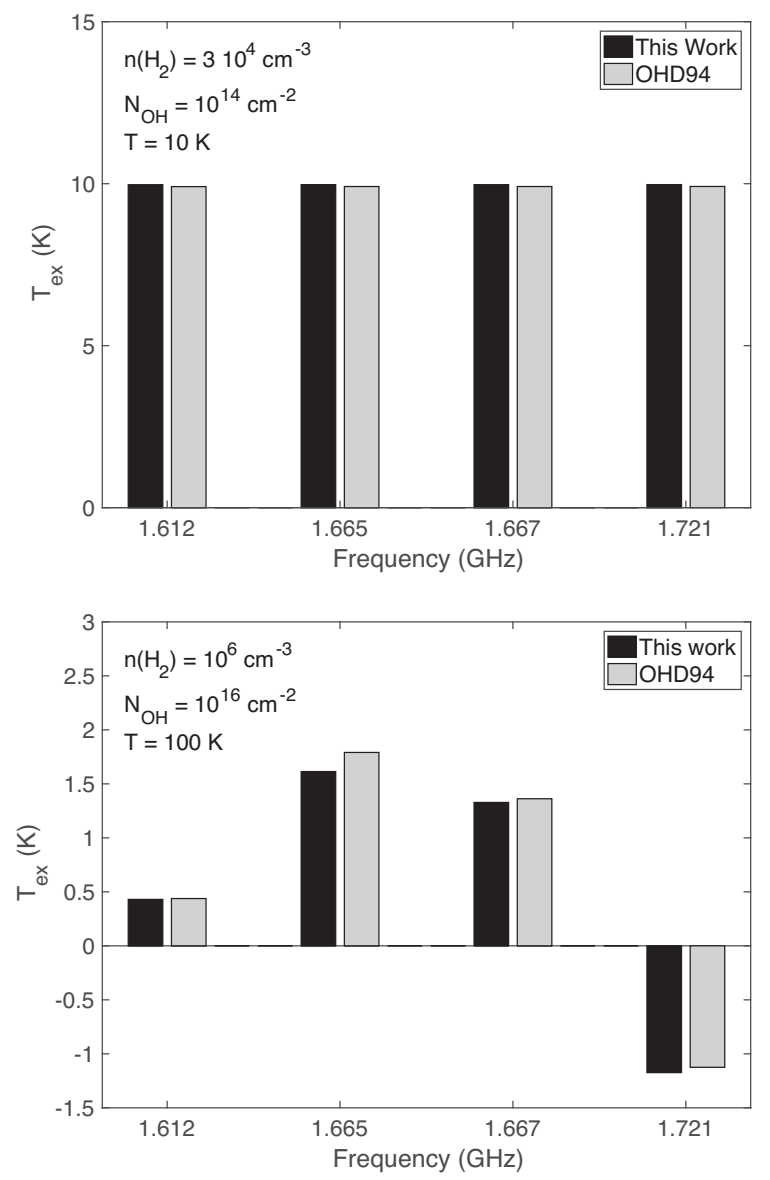

Figure 4. Excitation temperature (in $\mathrm{K}$ ) of the four hyperfine components of the line $3 / 2 F_{1},+\rightarrow 3 / 2 F_{1},-$ at $1.66 \mathrm{GHz}$ obtained with the new rate coefficients and OHD94 rate coefficients. See the text for details.

structure levels are known to be strongly deviating from a single excitation temperature assumption. Maser emission is even seen in some of the hyperfine components.

Hence, we consider a kinetic temperature of 10 and $100 \mathrm{~K}$, an $\mathrm{OH}$ column density of $10^{14}$ and $10^{16} \mathrm{~cm}^{-2}$, a linewidth of 1.0 and $5.0 \mathrm{~km} \mathrm{~s}^{-1}$, and a density of $3 \times 10^{4}$ and $10^{6} \mathrm{~cm}^{-3}$. These physical parameters correspond to the typical physical conditions in cold molecular clouds (Harju et al. 2000) and in low- and intermediatemass protostars and in star formation regions (Wampfler et al. 2011, 2013), respectively. We computed the excitation temperatures for the four hyperfine lines using the new rate coefficients and those of OHD94. Fig. 4 presents the results.

For the typical physical conditions of a cold molecular cloud, the excitation temperature of all these four lines approaches the kinetic temperature because of the very small corresponding Einstein coefficients $\left(<10^{-10} \mathrm{~s}^{-1}\right)$. No major differences are, thus, seen using one or the other set of collisional data because, in such physical conditions, LTE is a very good approximation, as already seen in paper I.

In contrast to what is found for cold molecular clouds, the excitation temperatures of the four lines are well below the kinetic temperature when considering the typical physical conditions of protostars and star formation regions. It is interesting to note that the excitation temperature is even below the CMB temperature for the lines at 1.612, 1.665, and 1.667 GHz. Both sets of collisional data predict a similar behaviour. For the line at $1.721 \mathrm{GHz}$, both sets of 


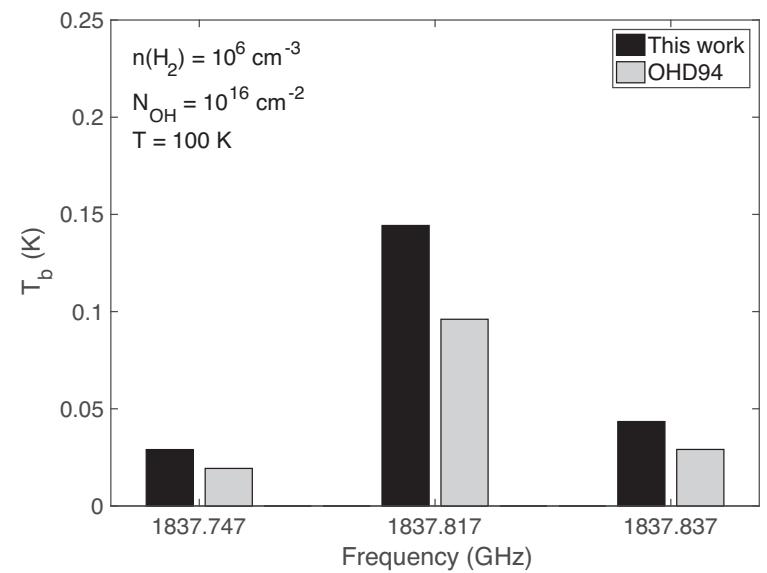

Figure 5. Brightness temperature (in $\mathrm{K}$ ) of the three hyperfine components of the line $3 / 2 F_{2},+\rightarrow 1 / 2 F_{2},-$ at $1837.8 \mathrm{GHz}$ obtained with the new rate coefficients and OHD94 rate coefficients. See the text for details.

collisional rate coefficients predict a negative excitation temperature so that maser emission is predicted for this component. We note that the excitation temperatures of the four lines are significantly different, showing evidence of non-uniform excitation conditions. The impact of the new rate coefficients versus the use of the previous ones is, however, moderate.

Next, we computed the brightness temperature for the three hyperfine components of the line $j=3 / 2 F_{2},+\rightarrow j=1 / 2 F_{2},-$ at $1837.8 \mathrm{GHz}$ using the new rate coefficients and those of OHD94. These hyperfine resolved transitions have been detected with Herschel/HIFI towards a star-forming region (Wampfler et al. 2011). Fig. 5 shows the results of our radiative transfer calculations.

We note that the line intensities obtained using the new rate coefficients are larger than those obtained using the OHD94 data, the line intensity ratios being $\simeq 1.5$. Such a difference is attributed to the difference between the two sets of collisional data. Indeed, the new rate coefficients are larger than those of OHD94 by a factor of 1.5-2. As a result, the present calculations show that for a given physical structure, the $\mathrm{OH}$ column density derived from radiative transfer analysis performed using OHD94 rate coefficients is overestimated by up to 50 percent. It is then crucial to use the new collisional data to revise the $\mathrm{OH}$ abundance in star-forming and protostellar regions.

Finally, we note that in their detailed study of the $\mathrm{OH} 18 \mathrm{~cm}^{-1}$ transition towards molecular clouds, Ebisawa et al. (2019) have checked the robustness of their non-LTE calculations (based on OHD94 data) by using the fine-structure rate coefficients of Paper I to which the (approximate) $M_{j}$ random method was applied. They concluded that the uncertainties in the OHD94 collisional rate coefficients 'have only a limited effect, a factor of 2 at most', in agreement with the present results.

\section{CONCLUSION}

We have computed a new set of hyperfine structure resolved rate coefficients for the $\mathrm{OH}-\mathrm{H}_{2}$ collisional system based on an accurate set of PESs combined with quantum close-coupling scattering calculations. The state-to-state rate coefficients were derived for the lowest 24 hyperfine levels of $\mathrm{OH}$, with both para- $\mathrm{H}_{2}\left(j_{2}=0\right)$ and ortho- $\mathrm{H}_{2}\left(j_{2}=1\right)$, in the temperature range $10-150 \mathrm{~K}$. The full set of data will be made available in the LAMDA (Schöier et al. 2005) and BASECOL (Dubernet et al. 2013) databases. We have found that the new $\mathrm{OH}-\mathrm{H}_{2}$ rate coefficients are significantly larger than those of Offer et al. (1994) currently used for astrophysical modelling. The new rate coefficients were found to increase the hyperfine intensities by a factor of $\sim 1-2$. Consequently, we recommend to use this new set of data in any astrophysical model of $\mathrm{OH}$ excitation.

\section{ACKNOWLEDGEMENTS}

We acknowledge financial support from the US National Science Foundation, Grant No. CHE-1565872. JK acknowledges XSEDE.org Grant No. CHE-130120 and University of Maryland High-Performance Deepthought facilities for computational time. FL and AF acknowledge the European Research Council (Consolidator Grant COLLEXISM, grant agreement 811363) and the financial support from the Institut Universitaire de France and from the Agence Nationale de la Recherche (ANR-HYDRIDES), contract ANR-12- BS05-0011-01.

\section{REFERENCES}

Alexander M. H., Dagdigian P. J., 1985, J. Chem. Phys., 83, 2191

Cotten D. L., Magnani L., Wennerstrom E. A., Douglas K. A., Onello J. S., 2012, AJ, 144, 163

Dubernet M. L. et al., 2013, A\&A, 553, A50

Ebisawa Y., Inokuma H., Sakai N., Menten K. M., Maezawa H., Yamamoto S., 2015, ApJ, 815, 13

Ebisawa Y., Sakai N., Menten K. M., Yamamoto S., 2019, ApJ, 871, 89

Goicoechea J. R. et al., 2011, A\&A, 530, L16

Groenenboom G. C., Fishchuk A. V., van der Avoird A., 2009, J. Chem. Phys., 131, 124307

Harju J., Winnberg A., Wouterloot J. G. A., 2000, A\&A, 353, 1065

Hibridon, 2012, is a package of programs for the time-independent quantum treatment of inelastic collisions and photodissociation written by M. H. Alexander, D. E. Manolopoulos, H.-J. Werner, B. Follmeg, P. J. Dagdigian, and others. More information and/or a copy of the code can be obtained from the website http://www2.chem.umd.edu/groups/alexa nder/hibridon

Huber K. P., Herzberg G., 1979, Molecular Spectra and Molecular Structure. IV. Constants of Diatomic Molecules. Van Nostrand Reinhold, New York, NY

Kłos J., Ma Q., Dagdigian P. J., Alexander M. H., Faure A., Lique F., 2017, MNRAS, 471, 4249 (Paper I)

Ma Q., Kłos, J. Alexander M. H., van der Avoird A., Dagdigian P. J., 2014, J. Chem. Phys., 141, 174309

Offer A., Flower D. R., 1990, J. Phys. B: At. Mol. Opt. Phys., 23, L391

Offer A. R., van Hemert M. C., 1993, J. Chem. Phys., 99, 3836

Offer A. R., van Hemert M. C., van Dishoeck E. F., 1994, J. Chem. Phys., 100, 362 (OHD94)

Roueff E., Lique F., 2013, Chem. Rev., 113, 8906

Schewe H. C. et al., 2015a, J. Chem. Phys., 142, 204310

Schewe H. C. et al., 2015b, J. Chem. Phys., 142, 204310

Schöier F. L., van der Tak F. F. S., van Dishoeck E. F., Black J. H., 2005, A\&A, 432, 369

van der Tak F. F. S., Black J. H., Schöier F. L., Jansen D. J., van Dishoeck E. F., 2007, A\&A, 468, 627

Wampfler S. F. et al., 2011, A\&A, 531, L16

Wampfler S. F. et al., 2013, A\&A, 552, A56

This paper has been typeset from a $\mathrm{T}_{\mathrm{E}} \mathrm{X} / \mathrm{IAT}_{\mathrm{E}} \mathrm{X}$ file prepared by the author. 